\title{
Development of an R Package for Syndromic Surveillance: Translating Research into Tools for the Veterinary Epidemiologist
}

\author{
Fernanda C. Dórea ${ }^{\star 1}$, Stefan Widgren ${ }^{1}$, Ann Lindberg ${ }^{1}$ and Crawford W. Revie ${ }^{2}$ \\ ${ }^{1}$ Swedish Zoonoses Centre, National Veterinary Institute, Uppsala, Sweden; ${ }^{2}$ Atlantic Veterinary College, Charlottetown, PE, Canada
}

\section{Objective}

To summarize the challenges in the development of syndromic surveillance tools in veterinary medicine, and describe the development of an R package to address some of the current gaps.

\section{Introduction}

A review of the development of veterinary syndromic surveillance in 2011 [1] indicated that the field was incipient, but fast growing. Many countries are starting to explore different sources of data for syndromic surveillance [2]. Some of the data streams evaluated share similarities with those used in public health syndromic surveillance, such as clinical records and laboratory data. However, many unique animal data sources have arisen, such as abattoir and carcass collection data.

We suggest there are three main challenges in the current development of animal syndromic surveillance:

- The lack of standards in disease classification;

- The development of statistical methods appropriate to deal with animal data;

- The creation of ready-to-use tools that employ these statistical methods.

\section{Methods}

The development of standards for animal disease classification is a complex issue, which remains in its infancy. The subject is the goal of parallel efforts in which our group is involved, and is being addressed by: collaborations with institutions from different countries, the support of the ISDS contributing their expertise from human health surveillance, and plans to further develop the subject in an upcoming conference among veterinary epidemiologists.

While these standards are being developed, we propose to create an $\mathrm{R}$ package that will cover the remaining steps in the process of converting data into epidemiological information through automated analysis of animal health data streams, as shown in the Figure. The grey box highlights the steps that the proposed $\mathrm{R}$ package would initially address. Next steps would include syndromic classification algorithms, as standards are agreed.

The proposed package will take advantage of object-oriented programming in R. As such, each data stream will be processed in order to add slots that directly associate to them statistical and epidemiological information resulting from the analysis. Alarm scores resulting from the application of multiple algorithms for detection of outbreak signals, for instance, would then become intrinsic parts of the object. Algorithms whose use has been documented in animal health, such as different types of control charts and Holt-Winters exponential smoothing, will be implemented.

Object oriented programming will facilitate the association of specific behaviors to common R functions such a "print" and "plot", allowing even novice $\mathrm{R}$ users to generate epidemiologically relevant summaries and plots, both before and after analyses.
Moreover, functions will be made available in the package to create an HTML-based interface, which could include maps of the recorded events if they are georeferenced.

\section{Conclusions}

$\mathrm{R}$ packages are available which support specific data analysis algorithms, but their application requires experienced users capable of incorporating these algorithms into the larger framework of a syndromic surveillance system. We propose a package in which various statistical analyses already tested for use in animal data streams will be available, coupled with data management capabilities and output visualization. The main innovation offered by the package will be the ability to manage data streams, analyses, alarms and user interface in a continuous flow; all contained in a single, open source, package. This will facilitate the implementation of syndromic surveillance systems by veterinary epidemiologists.

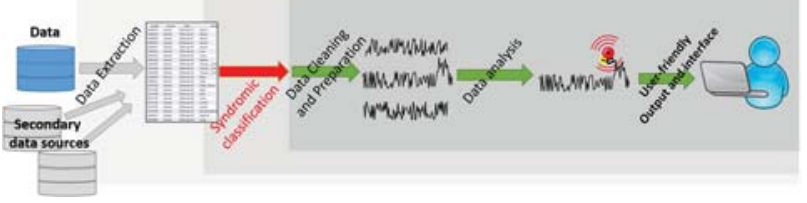

\section{Keywords}

animal health; syndromic surveillance; R programming

\section{References}

[1] Dórea, FC, Sanchez, J and Revie, CW. 2011. Veterinary syndromic surveillance: Current initiatives and potential for development. Prev Vet Med 2011 (101) 1-2 1-17.

[2] Dupuy C, Bronner A, Watson E, Wuyckhuise-Sjouke L, Reist M, Fouillet A, Calavas D, Hendrikx P, Perrin JB. Inventory of veterinary syndromic surveillance initiatives in Europe (Triple-S project): Current situation and perspectives. Prev Vet Med 2013 (111) 3-4 220-9

\section{*Fernanda C. Dórea}

E-mail: fernanda.dorea@sva.se 